\title{
Plastic accommodation during tensile deformation of gradient structure
}

\author{
Xiaolei $\mathrm{Wu}^{1,2^{*}}$, Muxin Yang ${ }^{1}$, Runguang $\mathrm{Li}^{3}$, Ping Jiang ${ }^{1}$, Fuping Yuan ${ }^{1,2}$, Yandong Wang ${ }^{3 *}$, \\ Yuntian $\mathrm{Zhu}^{5}$ and Yueguang $\mathrm{Wei}^{4^{*}}$
}

\begin{abstract}
Gradient structure (GS) possesses a typical trans-scale grain hierarchy with varying internal plastic stability, and the mutual plastic accommodation plays a crucial role in its superior strength-ductility combination. Using the in-situ synchrotron X-ray diffraction (XRD) during tensile loading, we measured lattice strains sequentially from the nanostructured (NS) surface layer to the central coarsegrained (CG) layer to elucidate when and how plastic accommodation occurs and evolves within the GS, along with their roles in plastic deformation and strain hardening. Throughout the tensile deformation, two types of plastic incompatibility occur in the GS. One is an extended elastoplastic transition due to layer-by-layer yielding. The other is strain localization and softening in the NS layer, in contrast with the stable plastic deformation in the CG layer. Plastic accommodation thus occurs concurrently and manifests as both an inter-layer and intra-layer change of stress state throughout tensile deformation. This produces different micromechanical responses between layers. Specifically, the NS layer initially experiences strain hardening followed by an elastoplastic deformation. The hetero-deformation induced hardening, along with forest hardening, facilitates a sustainable tensile strain in the NS layer, comparable to that in the CG layer.
\end{abstract}

Keywords: gradient structure, plastic accommodation, strain hardening, nanostructure, ductility

\section{INTRODUCTION}

Gradient structure (GS) is attracting ever-increasing attention as an effective strategy for achieving superior combination of strength and ductility in metallic materials [1-5]. The GS is usually composed of a nano- structured (NS) surface layers sandwiching a central coarse-grained (CG) layer, with gradient grain size distribution in-between $[3,4]$. Therefore, the GS can also be regarded as an integration of individual layers, with an outer-inner arrangement from the NS layer to CG layer [6]. Hence, the GS is a typical trans-scale grain hierarchy, usually spanning grain sizes of 3-4 orders of magnitude $[3,4]$.

The GS displays microscopically heterogeneous tensile deformation $[3,4,7,8]$, due to the significant difference between the two plastic responses of individual layers. In one response, the CG layer yields first followed by the remaining layers, depending on the grain size according to the classic Hall-Petch relationship $[9,10]$. This leads to gradual elastoplastic (e-p) transition [11-13]. Therefore, yielding in the GS should result in heterogeneous e-p codeformation, which has not been directly observed [14]. The other response is plastic localization in the NS layer, which promotes early necking due to strain localization $[3,4]$, while the CG layer remains stable. Accordingly, plastic incompatibility between individual layers is inherent to the GS and the origin of heterogeneous tensile deformation $[4,7,14]$.

Plastic accommodation occurs concurrently with heterogeneous deformation in the GS. Geometrically necessary dislocations (GNDs) [15-17] are generated to accommodate the strain difference across hetero-interfaces. The GNDs produce additional long-range internal stress and internal strain hardening [18-23], initially termed back stress hardening $[7,19,24]$ and now known as hetero-deformation induced (HDI) hardening $[8,14]$. The strain gradient, as a way for plastic accommodation of the

\footnotetext{
${ }^{1}$ State Key Laboratory of Nonlinear Mechanics, Institute of Mechanics, Chinese Academy of Sciences, Beijing 100190, China

${ }^{2}$ School of Engineering Science, University of Chinese Academy of Sciences, Beijing 100049, China

${ }^{3}$ State Key Laboratory for Advanced Metals and Materials, University of Science and Technology Beijing, Beijing 100083, China

${ }^{4}$ Department of Mechanics and Engineering Science, College of Engineering, BIC ESAT, Peking University, Beijing 100871, China

${ }^{5}$ Department of Materials Science and Engineering, City University of Hong Kong, Hong Kong, China

* Corresponding authors (emails: xlwu@imech.ac.cn (Wu X); ydwang@bust.edu.cn (Wang Y); weiyg@pku.edu.cn (Wei Y))
} 
GS, was measured $[4,25]$ and the HDI hardening was revealed at the hetero-interfaces between ductile CG layers and hard NS layers $[4,25]$. However, heterogeneous deformation and plastic accommodation are often coupled in the GS [25]. In fact, plastic accommodation occurs soon after yielding and continues through tensile deformation.

Plastic accommodation, therefore, plays a crucial role in producing superior mechanical properties [3-6,26-35], as an inevitable reflection of heterogeneous deformation in response to strengthening (yield strength, $\sigma_{y}$ ) and subsequent strain hardening [36]. It remains unclear how heterogeneous tensile deformation proceeds in the GS. More specifically, some may wonder when and how plastic accommodation occurs and evolves in the individual layers. The details of inter-layer and intra-layer interaction together with the micromechanical response of each layer are the focus of this study. In the present investigation, the synchrotron-based X-ray diffraction (XRD) in situ tensile loading was performed. Based on the layer-by-layer measurements, as a function of applied stress in the GS, the change of lattice strains was obtained to demonstrate the formation and evolution of plastic accommodation and inter-layer and intra-layer interaction during tensile deformation.

\section{EXPERIMENTAL SECTION}

\section{Materials and sample preparation}

The material used in this investigation was a body-centered-cubic (bcc) structured interstitial-free (IF) steel with a chemical composition (wt\%) of $0.003 \% \mathrm{C}, 0.08 \% \mathrm{Mn}$, $0.009 \% \mathrm{Si}, 0.008 \% \mathrm{~S}, 0.011 \% \mathrm{P}, 0.037 \% \mathrm{Al}, 0.063 \% \mathrm{Ti}$, $38 \mathrm{ppm} \mathrm{N}$, and the balance Fe. An original 1-mm-thick sheet was annealed at $1123 \mathrm{~K}$ for $1 \mathrm{~h}$ to form a homogeneous single-phase CG microstructure with a mean grain size of $26 \mu \mathrm{m}$.

The GS samples were processed using the surface mechanical attrition treatment (SMAT), see Refs $[37,38]$ for details. Briefly, the SMAT technique involves ultra-high strain-rate impacts of spherical shots (diameter of $4 \mathrm{~mm}$ ) on the sheet surface under high-power ultrasound waves $(20 \mathrm{kHz})$. The entire sheet surface was peened with many impacts over a short period of time. The gradient distribution appeared in both the strain and strain rate in the depth direction. This leads to an outer-inner grain refinement through progressive plastic deformation. A GS is thus generated consisting of various grain sizes from the NS surface layer towards the central CG layer. Each side of the sheet was subjected to SMAT processing for
$8 \min$.

\section{In-situ synchrotron-based XRD during tensile loading}

The in situ synchrotron XRD during tensile loading was carried out on the beam-line 11-ID-C at the Advanced Photon Source (APS), Argonne National Laboratory, USA. Fig. 1a shows a schematic of the experimental setup. The details can be found in the Refs $[39,40]$. A monochromatic X-ray beam, with an energy of $105.1 \mathrm{keV}$ at a wavelength of $0.117418 \AA$, was used to map the structural characteristics. The X-ray beam was oriented perpendicular to the side of GS sheet, as indicated in Fig. 1a. A two-dimensional (2D) detector was placed $1800 \mathrm{~mm}$ behind the tensile sample to collect the intensity data of the diffraction rings. The slit size (i.e., beam size) was $500 \mu \mathrm{m}$ by $100 \mu \mathrm{m}$. XRD measurements
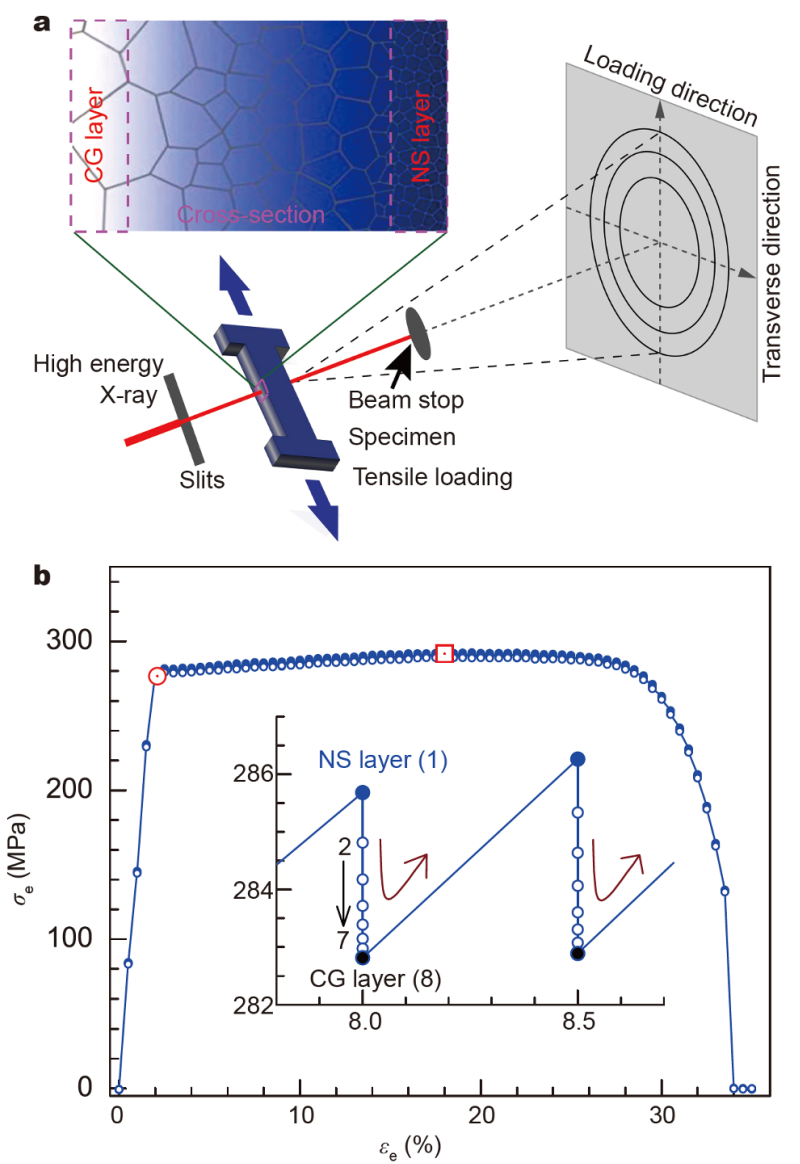

Figure 1 In-situ synchrotron-based XRD during tensile loading of GS. (a) Schematic set-up for layer-by-layer XRD measurements from NS surface layer to central CG layer. (b) Tensile engineering stress-strain $\left(\sigma_{\mathrm{e}}-\mathcal{E}_{\mathrm{e}}\right)$ curve, tested in situ. Inset: an enlargement of the curve segment. Each circle indicates a single measurement. Layer 1 is the NS layer, while layer 8 is the CG layer. A total of eight measurements were performed at each strain level. 
were conducted as the GS sample was subjected to increasing strain. The crystallographic plane was determined from the diffraction patterns and then lattice strains were calculated based on the change in the measured inter-planar spacing, using $\left(d^{h k l}-d_{0}^{h k l}\right) / d_{0}^{h k l}$, where $d_{0}^{h k l}$ is the $\mathrm{d}$-spacing of the $(h k l)$ grain family before initial applied stress. Therein, $d_{0}$ was determined from an annealed CG sample before tensile deformation.

The GS sample used for tensile testing was dog-bone shaped. Its gauge section was $16.15 \mathrm{~mm}$ long, $1.13 \mathrm{~mm}$ wide, and $\sim 0.9 \mathrm{~mm}$ thick. The engineering strain was measured from cross-head displacement. Synchrotron $\mathrm{XRD}$ was conducted layer-by-layer in the depth direction of the GS sample (i.e., gradient direction) to determine the change in lattice strain of each individual layer during tensile loading. The in-situ loading was strain-controlled, conducted from the outside inwards, and repeated eight times in the depth direction, with a scanning step of $100 \mu \mathrm{m}$, loading step of $50 \mu \mathrm{m}$, and exposure time of $1 \mathrm{~s}$. The in situ measured tensile engineering stress-engineering strain $\left(\sigma_{\mathrm{e}}-\varepsilon_{\mathrm{e}}\right)$ curve is shown in Fig. 1b. The inset shows the loading detail. Each circle represents one measurement during which straining is on hold. Layer 1 is the NS layer, while layer 8 is the CG layer. The width of each layer, i.e., slit width, is $100 \mu \mathrm{m}$, with an overlapping width of $50 \mu \mathrm{m}$. The interval of strain level was $0.5 \%$ for each measurement. A total drop in flow stress of $\sim 5 \mathrm{MPa}$ appears during the eight measurements, with little effect on plastic deformation.

\section{Transmission electron microscopy observations}

The microstructural characterizations of GS samples were conducted using transmission electron microscopy (TEM). The cross-sectional TEM films were cut at varying depths from a gauge section of the tensile samples.

\section{RESULTS}

\section{Microstructure of the GS}

Fig. 2a shows the cross-sectional morphology of nearly half of the thickness of the GS sample. The NS surface layer and CG layer are located on the left and right sides, respectively. The electron backscatter diffraction (EBSD) image (inset) shows the recrystallized CG layer with an average grain size of $26 \mu \mathrm{m}$. TEM images reveal the microstructures at varying depths, i.e., nano-grains (Fig. 2b) and ultrafine-grains (Fig. 2c) in the NS layer, elongated sub-grains (Fig. 2d) and dislocation cells and tangles (Fig. 2e) in the deformed layer. Both the grain size and
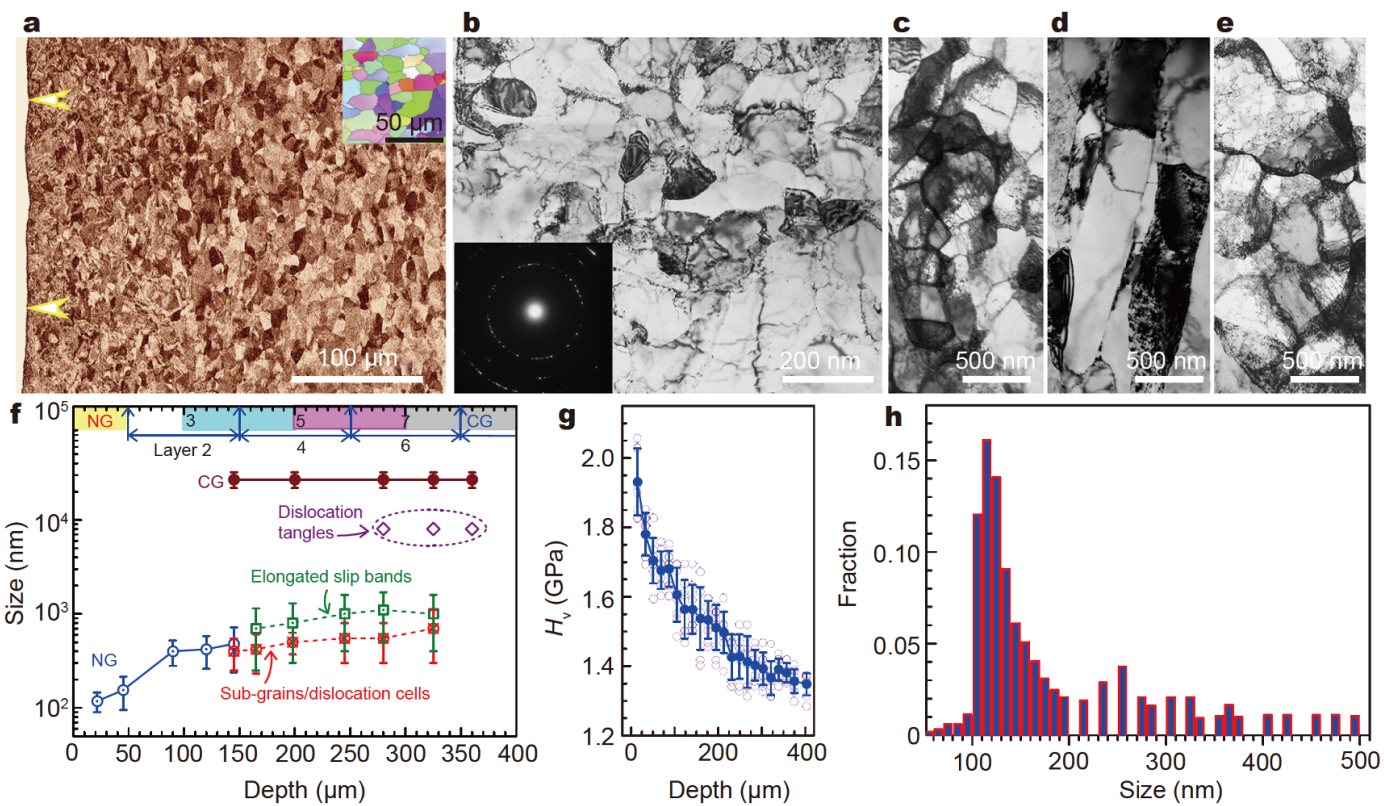

Figure 2 Microstructure of the GS. (a) Transverse optical morphology nearly half the thickness of the GS sample. Arrows: top NS surface layer. Inset displays the electron back scatter diffraction image of the central CG layer. (b-e) Typical bright-field TEM images taken from depths of 22, 45, 165, and $280 \mu \mathrm{m}$ below the sample surface, showing nano-grains (b), ultrafine grains and subgrains (c), elongated slip bands (d), and dislocation tangles and cells (e), respectively. Inset in (b): the selected area electron diffraction pattern. (f) Gradient grain size distribution. The numbers indicate the positions of the individual layers that were subjected to XRD analysis. Note that the thicknesses of all the layers were $100 \mu \mathrm{m}$, except for the NS layer of $50 \mu \mathrm{m}$. (g) $H_{v}$ distribution of GS sample in the depth direction from the treated surface. (h) Grain size distribution in the top $22-\mu \mathrm{m}-\mathrm{deep}$ layer. 
microhardness $\left(H_{\mathrm{v}}\right)$ appear graded toward the center, see Fig. 2f, g, respectively. The surface NS layer is $\sim 50 \mu \mathrm{m}$ thick with a mean grain size of $110 \mathrm{~nm}$ (Fig. 2h), without any detectable growth during tensile loading [25]. Obviously, this GS is trans-scale grained, with grain sizes spanning three orders of magnitude.

\section{Tensile deformation in GS}

Fig. 3a, b show the change in XRD intensity with applied strain, $\varepsilon_{\text {app }}$, in the NS and CG layers, respectively. Clearly, the diffraction intensity of the (110) grain family is at least an order of magnitude higher than that of the (200) and (211) grain families in both layers. This is probably due to the SMAT processing of bcc grains [37]. Next, comparative analysis of lattice strain evolution in two representative layers was conducted, i.e., the strongest NS and softest CG layers, to reveal inter-layer and intra-layer plastic accommodation during tensile deformation of the GS.

\section{Layer-by-layer yielding}

Based on the synchrotron XRD measurements, the volume-averaged axial (i.e., parallel to tensile axis) and transverse elastic lattice strain, labeled $\varepsilon_{11}$ and $\varepsilon_{22}$, respectively, were calculated for the $(h k l)$ grain families of each layer as a function of applied stress, $\sigma_{\text {app }}$ or $\varepsilon_{\text {app}}$, together with the full width at half maximum (FWHM) values of the diffraction peaks.

Fig. 4a shows the change of both $\varepsilon_{11}$ and $\varepsilon_{22}$ of the (110) grain family with $\sigma_{\text {app }}$ in the NS and CG layers, respectively. Several $\varepsilon_{\text {app }}$ values corresponding to characteristic plastic responses are labeled, see $Y$-axis on the right side. Two stages, namely yielding and uniform deformation, can be identified in terms of $\sigma_{\mathrm{y}}$ of $280 \mathrm{MPa}$, from the $\sigma_{\mathrm{e}^{-}}$ $\varepsilon_{\mathrm{e}}$ curve (Fig. 1b).
The CG layer yields first. This micro-yielding in the CG layer is the starting point of deviation from the initial linearity of $\varepsilon_{11}$. The micro-yield stress $\left(\sigma_{\mathrm{my}}\right)$ is thus determined to be $145 \mathrm{MPa}$ at $\varepsilon_{\text {app }}$ of $1 \%$. Once microyielding occurs, two kinds of plastic accommodation immediately start. One is a change of stress state $[4,6,7,41]$, evidenced by the reversal of the sign of the slope of $\varepsilon_{22}$ in the CG layer, see the line segment indicated by an arrow in Fig. 4a. This reversal signals a redirection of lateral compressive stress, instead of a decrease in magnitude of $\varepsilon_{22}$ [41]. It is the result of inter-layer interaction due to the e-p incompatibility by the difference in apparent Poisson's ratio between the plastically deformed CG layer $(v=0.5)$ and still elastic NS layer [6,4244]. The second is load partitioning, an inevitable result of the change of stress state. The rate of increase of $\varepsilon_{11}$ decreases in the CG layer, deviating from the initially linear $\varepsilon_{11}$ to the left, while this rate increases in the NS layer, tilting from the linear $\varepsilon_{11}$ to the right, see curved arrows in Fig. 4a. Accordingly, the CG layer bears less $\sigma_{\text {app }}$, while the NS layer bears a proportionally increasing fraction of $\sigma_{\text {app }}$. Load partitioning is the typical plastic response of composite-like structures, often occurring in composites, dual-phase structures, and grain hierarchies [41,45-48]. Hence, both the stress state change and load partitioning are ascribed to the plastic incompatibility between individual layers with different yield strengths.

The release of residual stress begins as $\sigma_{\text {app }}$ increases. Residual stress was introduced in the GS during SMAT processing [37] and was determined, in this GS sample, to be tensile in the CG layer and compressive in the NS layer [49]. Within the $\sigma_{\text {app }}$ range of 235 to $290 \mathrm{MPa}$, corresponding to $\varepsilon_{\text {app }}$ range of $1.5 \%$ to $3 \%$, all layers demonstrate irregular changes in $\varepsilon_{11}$, see Fig. 4a. For example, the initial change in $\varepsilon_{11}$ of the CG layer is insignificant
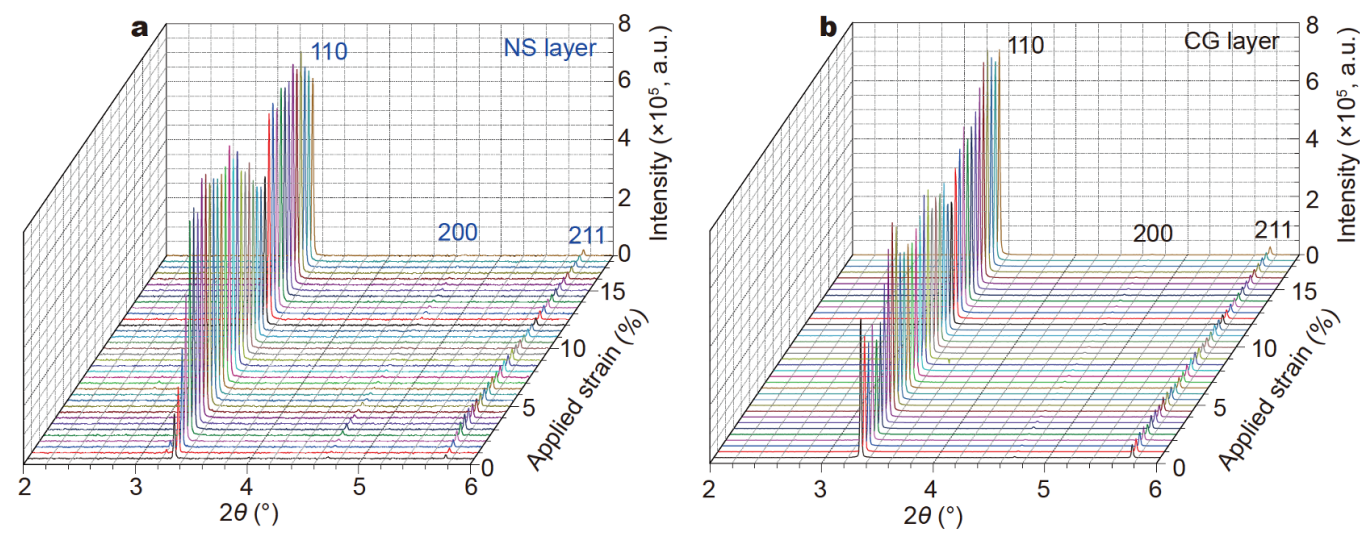

Figure 3 Change of XRD intensities of (110), (200), and (211) grain families with applied strain in the NS (a) and CG (b) layers. 

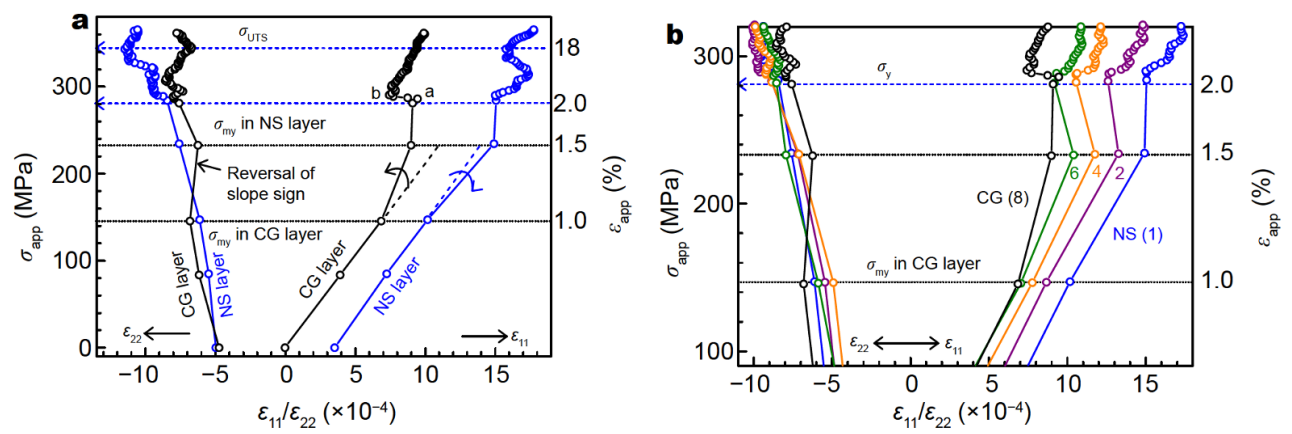

Figure 4 Evolution of lattice strains. (a) Change of both axial $\left(\varepsilon_{11}\right)$ and transverse lattice strain $\left(\varepsilon_{22}\right)$ in (110) grain family with applied stress (left $Y$ axis) and strain (right $Y$-axis) in both the NS and CG layers. Ultimate tensile strength $\left(\sigma_{\mathrm{UTS}}\right)$ and $\sigma_{\mathrm{y}}$ are of 296 and $280 \mathrm{MPa}$ in GS sample. (b) The details of the lattice strains below $\sigma_{y}$, along with those of three more layers.

before dropping dramatically from point a to b. This indicates the release of in-plane, axial residual stress $[50,51]$. The NS layer also shows little change in $\varepsilon_{11}$. Interestingly, $\varepsilon_{11}$ increases again in all layers when $\sigma_{\text {app }}$ exceeds $290 \mathrm{MPa}$, slightly higher than $\sigma_{\mathrm{y}}$. This represents the point at which the release of residual stress is essentially complete, consistent with previous results where the release of residual stress ends soon after yielding occurs [50].

Yielding in a GS is progressive micro-yielding in individual layers. Fig. $4 \mathrm{~b}$ shows the details of $\varepsilon_{11}$ and $\varepsilon_{22}$ during the yielding stage, including the results of three more layers. Refer to Fig. $2 \mathrm{f}$ for the specific location of each layer. Layer 6, close to the CG layer, demonstrates a lower rate of increase in $\varepsilon_{11}$ than the CG layer, even with micro-yielding seemingly still at $\varepsilon_{\text {app }}$ of $1 \%$. This suggests a later onset of micro-yielding compared with the CG layer. Layer 2 looks like the NS layer (layer 1), i.e., it has similar load distribution. Yet, the middle layer (layer 4) maintains a linear $\varepsilon_{11}$, even without the involvement of load distribution. Hence, the GS yields layer-by-layer, even if micro-yielding is hardly definitive in the NS layer and layer 2 due to the interference of residual stress release. Accordingly, a conservative estimate of $\sigma_{\mathrm{my}}$ is $240 \mathrm{MPa}$ at $\varepsilon_{\text {app }}$ of $1.5 \%$, see Fig. $4 \mathrm{~b}$, corresponding to the onset of stress release. Therefore, the GS experiences an extended e-p co-deformation, as large as $1 \%$ arising from the micro-yielding in the CG layer, at $\varepsilon_{\text {app }}$ of $1 \%$, to universal yielding at $\varepsilon_{\text {app }}$ of $2 \%$.

Three grain families in each layer show varying responses during yielding in the GS. Fig. 5 shows the change of both $\varepsilon_{11}$ and $\varepsilon_{22}$ in (110), (200), and (211) grain families with $\sigma_{\text {app }}$, respectively, of the NS and CG layer. Clearly, the responses of both $\varepsilon_{11}$ and $\varepsilon_{22}$ differ greatly between the three grain families. The NS layer shows the
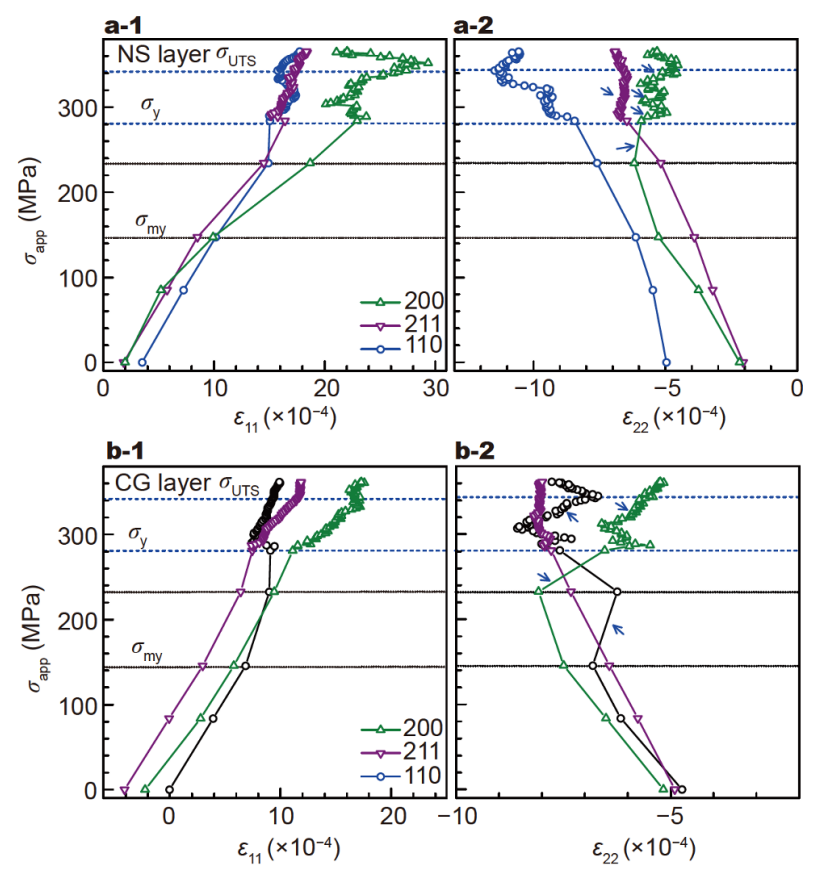

Figure 5 Change of both $\varepsilon_{11}$ (left) and $\varepsilon_{22}$ (right) with $\sigma_{\text {app }}$ in the (110), (200), and (211) grain families in the NS (a) and CG (b) layers, respectively. All arrows in $\varepsilon_{22}-\sigma_{\text {app }}$ curves indicate the reversal of the sign of the slope.

fastest rate of increase in $\varepsilon_{11}$ for the (200) grain family, followed by the (211) and the (110) grain families (Fig. 5a1). It is, therefore, the (200) grain family that bears the greatest load in the NS layer and, thus, will yield last. In comparison, micro-yielding in CG layer occurs (Fig. 5b1), in the (110) grain family first, then in the (211) grain family at $\sigma_{\text {app }}$ of $225 \mathrm{MPa}\left(\varepsilon_{\text {app }}\right.$ of $\left.1.5 \%\right)$, and finally in the (200) grain family at $\sigma_{\text {app }}$ of $280 \mathrm{MPa}\left(\varepsilon_{\text {app }}\right.$ of $\left.2 \%\right)$. Both the CG and NS layers, therefore, show extended micro-yielding due to elastic anisotropy of different grain 
families. Moreover, the reversal of the sign of the slope of $\varepsilon_{22}$ is unique to the (211) grain family in both the NS and CG layers during micro-yielding, as indicated by arrows in Fig. 5a2, b2. This indicates an intra-layer change of stress state. Both inter-layer and intra-layer changes of stress state accompany the load re-distribution as soon as micro-yielding occurs.

\section{Plastic accommodation}

The GS starts to uniformly deform after the residual stress has been released. Fig. 6 details the changes in both $\varepsilon_{11}$ and $\varepsilon_{22}$ of the (110) grain family with $\sigma_{\text {app }}$, together with the FWHM value (as a measure of dislocation density $\rho$ ), from $\sigma_{\mathrm{y}}$ to ultimate tensile strength $\left(\sigma_{\mathrm{UTS}}\right)$ in both the NS and CG layers. The simultaneous rises in $\varepsilon_{11}$ and $\rho$ with $\sigma_{\text {app }}$ occur from $\varepsilon_{\text {app }}$ of $3 \%$ (point A) to $9 \%$ (point B) in the NS layer, see Fig. 6a1. This corresponds to uniform strain $\left(\varepsilon_{\mathrm{u}}\right)$ of $6 \%$ under conventional forest dislocation hardening. In the CG layer, the steady rise also occurs until $\sigma_{\mathrm{UTS}}$ at $\varepsilon_{\text {app }}$ of $18 \%$ (Fig. 6b1). Therefore, $\varepsilon_{\mathrm{u}}$ is $15 \%$ in CG layer. Note the drastic decrease in $\varepsilon_{11}$ from point B to $\sigma_{\mathrm{UTS}}$ in the NS layer, lasting a strain interval of $9 \%$. This drop indicates softening due to strain localization by shear banding in the NS layer [25]. The final random change of $\varepsilon_{11}$ is probably due to the severe plastic instability. Plastic strain, therefore, consists of $\varepsilon_{\mathrm{u}}$ of $6 \%$ and unstable strain of $9 \%$ in the NS layer.

Several features of plastic accommodation are apparent. First, the slope, i.e., the rate of increase of $\varepsilon_{11}$ in the NS layer, is 2.5 times that in the CG layer during uniform deformation of both layers. This is still the result of load distribution. Second, this rate in the NS layer accelerates, much faster in the (200) grain family than in both the (110) and (211) families, see Fig. 5a1. This indicates intralayer heterogeneous deformation within the NS layer. The same happens in the CG layer, see Fig. 5b1. The (200) grain family bears the greatest load, while the (110) family bears the largest plastic strain. Both the inter-layer and intra-layer plastic accommodation continues until uniform tensile deformation.

Strain localization is noteworthy in the NS layer. In-situ digital-image-correlation (DIC) during tensile testing of the same GS sample [25] revealed that a shear band nucleated soon after yielding in the NS layer and later expanded to the entire gauge section. In the present in situ test, the $0.5 \mathrm{~mm}$ long slit is very small, compared with the gauge length of $\sim 16 \mathrm{~mm}$ in the tested GS sample. The chance is too small for the beam to target the exact location where the shear-band nucleated. Here it was certainly not the case. Otherwise, strain softening would have occurred first [25].

The present result offers an opportunity to demonstrate
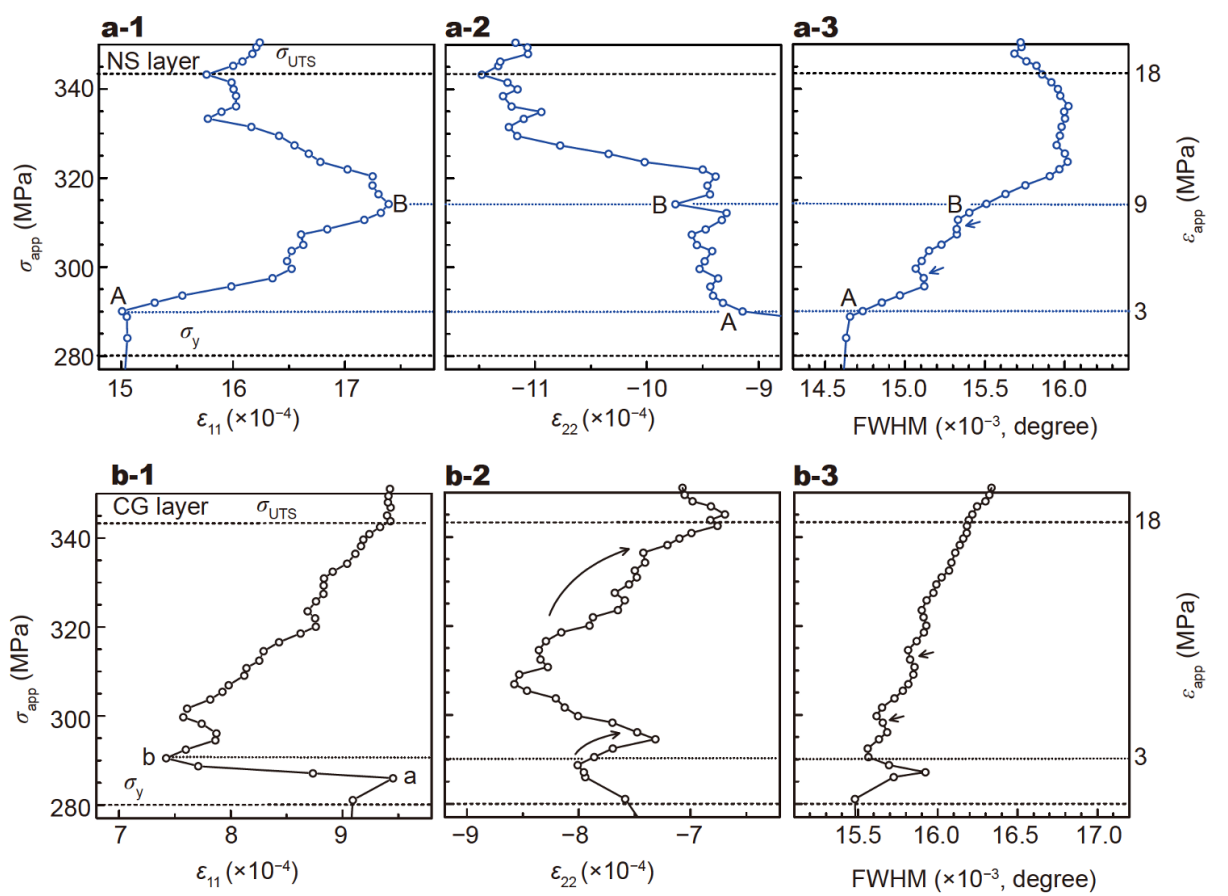

Figure 6 The details of lattice strain changes, along with the FWHM value, of (110) grain family from $\sigma_{\mathrm{y}}$ to $\sigma_{\mathrm{UTS}}$ in the NS (a1-a3) and CG (b1-b3) layers. 
the entire process of heterogeneous tensile response at the different locations within the NS layer. Importantly, specific plastic accommodation corresponds with strain localization. At this point, the reversal of the sign of the slope of $\varepsilon_{22}$ reoccurs in the CG layer, see arrows in Fig. $6 \mathrm{~b} 2$, in contrast to the trend observed in the NS layer (Fig. 6a2). This still indicates the change of stress state, similar to that previously observed during the e-p transitions, see Fig. 4a. Further, both the (200) and (211) grain families in the NS layer (Fig. 5a2), together with all three families in CG layer (Fig. 5b2), show the change of stress state, see arrows which indicate the reversal of the sign of the slope of $\varepsilon_{22}$. The origin of the stress state change is due to either the inter-layer plastic incompatibility between layers or intra-layer plastic incompatibility between three grain families. Strain localization leads to lateral shrinkage in the NS layer, much faster than in the CG layer $[4,25]$. For plastic accommodation, the CG layer will thus exert lateral tensile stress on the NS layer and as a result, the CG layer bears a balanced tensile stress. The intra-layer change of stress state occurred due to plastic incompatibility between grain families for the same reason.

\section{Evolution of dislocation density}

The change of FWHM value (equivalent to dislocation density $\rho$ ) offers further insight into plastic accommodation. In the CG layer, $\rho$ rises monotonously during tensile deformation, see Fig. 6b3. By comparison, $\rho$ drops twice in the NS layer. The first drop occurred during the e-p transition [49], and the second during the strain localization, see Fig. 6 a3.

The change of stress state is assumed to be responsible for the initial drop of $\rho[4,25]$. The change of stress state is equivalent to the transition of the strain route during multiple plastic deformation [52,53]. Upon changing the strain route, new slip systems are activated and the previously formed dislocations may disentangle and get annihilated $[4,25,52,53]$. As a result, $\rho$ decreases. During tensile deformation, the drop in $\rho$ in the NS layer responds to the change of stress state. Further, the propagating shear band also leads to the local change of stress state at the band front [54]. This explains the presence of a few intermittent drops in $\rho$, labeled by arrows in Fig. 6a3. It is argued that these intermittent drops of $\rho$ are the immediate response to the change of stress state. Moreover, the shear band develops at a high local strain rate, which expedites dislocation annihilation and leads to strain softening corresponding to a drastic drop in $\rho$ [25]. Further, this trend of $\rho$ in the NS layer agrees with pre- vious TEM observations [4]. Recently, the initial annihilation and subsequent generation of dislocations is evidenced in the shear band of NS layer through sitespecific TEM observations [25]. The drop in $\rho$ accommodates the newly formed dislocations. This is the reason for the rise of $\rho$ even to an $\varepsilon_{\text {app }}$ of $11 \%$, see Fig. 6a3. This also indicates effective forest dislocation hardening in the NS layer during tensile deformation.

\section{Strain hardening in NS layer}

Microscopically, each layer in the GS is in a multi-axial stress state due to the change of stress state, even under applied uniaxial tensile loading. The stress-strain response in each layer was thus analyzed using Equations $(1-3)$, where stress is the equivalent von Mises stress $\left(\sigma_{\mathrm{vM}}\right)$ [55]:

$\sigma_{\mathrm{vM}}=\sqrt{\sigma_{11}^{2}+\sigma_{22}^{2}-\sigma_{11} \sigma_{22}}$

$\sigma_{11}=\frac{E}{1-v^{2}}\left(\varepsilon_{11}+v \varepsilon_{22}\right)$,

$\sigma_{22}=\frac{E}{1-v^{2}}\left(\varepsilon_{22}+v \varepsilon_{11}\right)$,

where $\sigma_{11}$ and $\sigma_{22}$ are the principal axial and transverse stresses, $E$ is the elastic modulus and $v$ is Poisson's ratio. Here, $\sigma_{33} \approx 0$ since $\sigma_{33}$ is much smaller than $\sigma_{11}$ and $\sigma_{22}$ due to the much less constraint for free surface in the depth direction.

Fig. 7a shows the $\sigma_{\mathrm{vM}}$-true $\varepsilon_{\text {app }}$ curves of the NS and CG layers. Several other curves are also shown for comparison. The NS layer shows uniform strain up to $9 \%$ followed by near-ideal e-p deformation [56]. This contrasts sharply with the single NS layer which becomes unstable soon after yielding. Further, the principal transverse stress, $\sigma_{22}$, rises during the e-p transition and strain localization, respectively, in the NS layer, due to the change of stress state for plastic accommodation. The GS, along with the CG layer, shows continuous strain hardening. Fig. $7 \mathrm{~b}$ shows the strain hardening rate $(\Theta=\partial \sigma / \partial \varepsilon)$ versus true strain curves. $\Theta$ in the single NS layer plunges due to stain localization (necking). Conversely, the NS layer in the GS shows an up-turn in $\Theta$ soon after yielding, peaking at $\varepsilon_{\text {app }}$ of $\sim 4 \%$ followed by a slow drop. Note that $\Theta$ is lower than $\sigma_{\mathrm{vM}}$ from $\varepsilon_{\mathrm{app}}$ of $\sim 9 \%$. In fact, plastic instability occurs already in the NS layer in accordance with the Consideré criterion. This implies the crucial role of plastic accommodation. As a comprehensive response of integrated layers, $\Theta$ in the GS shows an up-turn during the e-p transition followed by a slow drop until final necking. Fig. 7c compares the $\sigma_{\mathrm{vM}}-\sigma_{\text {app }}$ curves in both the NS and CG layers. Usually, $\sigma_{\mathrm{vM}}$ is equal to $\sigma_{\text {app }}$ under 

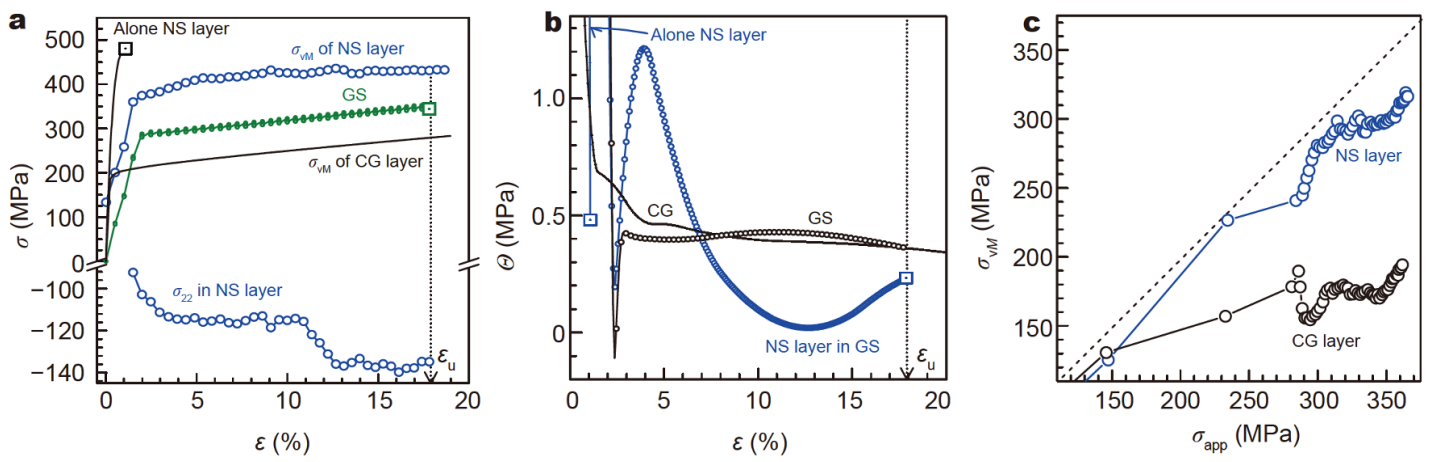

Figure 7 Micromechanical responses of the NS and CG layers. (a) Stress-strain curves; (b) strain hardening rate $(\Theta=\partial \sigma / \partial \varepsilon) v s$. true strain curves. Square: uniform tensile strain. (c) $\sigma_{\mathrm{vM}}-\varepsilon_{\text {app }}$ curves of the NS and CG layers, respectively.

uniaxial loading. The more $\sigma_{\mathrm{vM}}$ deviates from $\sigma_{\mathrm{app}}$, the more complicated the stress state. Clearly, $\sigma_{\mathrm{vM}}$ begins to deviate from $\sigma_{\text {app }}$ in both the CG and NS layers when the change of stress state occurs during micro-yielding and strain localization. Further, the deviation of $\sigma_{\mathrm{vM}}$ in the CG layer is greater than that in the NS layer. This indicates a more extreme change of stress state in CG layer, due to much larger $\sigma_{11}$, than in the NS layer (Fig. 7a).

\section{DISCUSSION}

Tensile deformation in the GS is microscopically heterogeneous due to different plastic responses among layers. The change of stress state, a crucial mechanical response, is typical of plastic accommodation, which plays a significant role in tensile deformation and strain hardening.

\section{Tensile deformation in GS}

The GS exhibits unique tensile deformation. Firstly, the GS yields layer-by-layer. The extended e-p strain is as large as $1 \%, 5$ times greater than conventional offset strain, i.e., $\sim 0.2 \%$, at which yield stress is defined for a uniform microstructure. Secondly, tensile responses differ not only between layers but also between different locations within layers. In contrast to the plastically stable CG layer, strain localization occurs in the NS layer and then intensifies until necking occurs. Strain localization leads to softening. Finally, both inter-layer and intra-layer plastic incompatibilities exist during tensile deformation. This is evidenced, respectively, by the reversal of the sign of the slope in $\varepsilon_{22}$ in either the CG layer or varying grain families (Figs $4 \mathrm{a}$ and $5 \mathrm{a} 2, \mathrm{~b} 2$ ) as well as opposite change in $\varepsilon_{11}$ of the three grain families (Fig. 5a1, a2). Plastic accommodation thus occurs in response to these plastic incompatibilities.

It has been long inferred from the stress-strain curve
(Fig. 1b), that each layer in the GS bears an equal $\varepsilon_{\mathrm{u}}$ such that tensile deformation is uniform in all layers $[3,4]$. This is plausible under uniaxial tensile load with both an isostrain and iso-strain rate. This assumption is flawed. First, $\varepsilon_{\mathrm{u}}$ differs in each layer, see Fig. 7a, and the apparent $\varepsilon_{\mathrm{u}}$ of the GS (Fig. 1b), combines the real $\varepsilon_{\mathrm{u}}$ for each layer. Second, the strain, together with the strain rate, is inhomogeneous between locations within the NS layer [25]. This is ascribed to the generation of a localized strain band and its subsequent propagation. The shear band experiences more strain, together with a faster strain rate [25]. Fig. 8 compares two curves to show the difference of tensile deformation in both the GS and NS layers. One is the $\sigma_{\text {app }}-\mathcal{E}_{\text {app }}$ curve of the GS layer (i.e., $\sigma_{\mathrm{T}}-\mathcal{E}_{\mathrm{T}}$ (subscript T stands for tensile) curve as shown in Fig. 7a), while the other is the $\sigma_{\mathrm{app}}-\varepsilon_{11}$ curve of the NS layer. For the GS, tensile response begins at $\sigma_{\text {my }}$ corresponding to $\varepsilon_{\text {app }}$ of $1 \%$ in the CG layer, followed by $\sigma_{\mathrm{y}}$ at $\varepsilon_{\text {app }}$ of $1 \%$. During this extended yielding, residual stress is released until $\varepsilon_{\text {app }}$

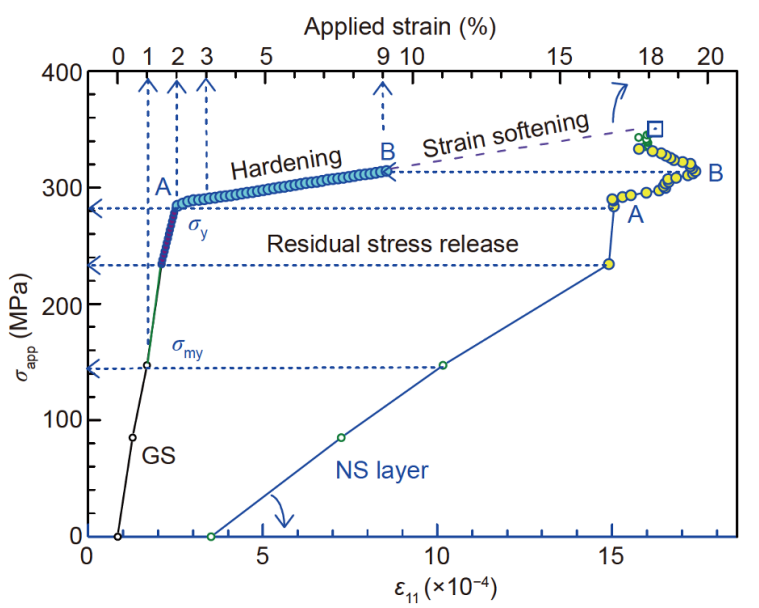

Figure 8 Comparison of $\sigma_{\text {app }}-\varepsilon_{\text {app }}$ and $\sigma_{\text {app }}-\varepsilon_{11}$ curves of the GS sample and NS layer. 
reaches 3\%. Later, the GS plastically deforms under strain hardening until necking occurs at $\varepsilon_{\text {app }}$ of $18 \%$ and $\varepsilon_{\mathrm{u}}$ of $15 \%$. However, $\varepsilon_{\mathrm{u}}$ in the NS layer is only $6 \%$ between $\varepsilon_{\text {app }}$ of $3 \%$ (point $A$ ) and $9 \%$ (point B). Strain localization happens at $\varepsilon_{\text {app }}$ of $9 \%$ (i.e., point B), which continues until necking occurs.

\section{Strain hardening}

Heterogeneous deformation causes HDI stress [8]. HDI stress involves back stress in the soft layer and forward stress in the hard layer $[8,14]$, which jointly induce HDI hardening [8]. The model of pile-up of GNDs against interfaces was proposed for HDI hardening [8,57-59]. Several key features of HDI hardening in the GS are noted. First, HDI hardening is associated with heterointerfaces, which has widespread distribution in the GS. This is most effective in suppressing strain localization in the entire NS layer. In comparison, HDI hardening locally affects grain boundaries of discrete CG layers with a bimodal grain structure [60]. Second, back stress induces strain hardening in the CG layer, with simultaneous rises in $\varepsilon_{11}$ and $\rho$ with $\sigma_{\text {app }}$. In contrast, forward stress induces softening in the NS layer, as shown by the decrease in $\varepsilon_{11}$ with $\sigma_{\text {app. }}$. Finally, HDI hardening stabilizes the NS layer which already displays plastically instability prior to diffuse necking. Therefore, HDI hardening is more effective during the later stage of tensile deformation, especially Stage-III hardening, when dynamic recovery weakens the effect of forest hardening. Forest dislocation hardening also affects the GS. This is due to the change of stress state, which leads to the drop in initial dislocations of high-density in the NS layer. In other words, the intragranular dislocation plasticity returns.

Moreover, the GS shows distinct synergistic strengthening $[5,6]$. Yield strength of the GS is greater than that predicted by the rule-of-mixture [6]. Extra strengthening was observed in varying GS [5,6]. The synergistic strengthening is also ascribed to HDI hardening which starts during the e-p co-deformation [61].

Plastic accommodation is an adaptive response which coincides with heterogeneous tensile deformation. The origin is the inter-layer and intra-layer plastic incompatibilities due to localized plastic stability in the trans-scale GS. The particular role of plastic accommodation is reflected in large plastic strains in the NS layer producing minimal strain hardening even under iso-strain and iso-strain rate tensile deformations. Synchrotron XRD analysis during in situ tensile loading enables the demonstration of the micromechanical response of individual layers to reveal the resultant inter-layer and intra-layer interactions and advance understanding of plastic deformation and strain hardening in the GS. Here, based on the in-situ measurements of layer-by-layer lattice strains, when and how plastic accommodation occurs and evolves in individual layers, together with the key effects on strain hardening, are elucidated.

\section{CONCLUSION}

Several key conclusions are drawn. First, the GS shows both inter-layer and intra-layer plastic incompatibilities throughout the tensile deformation process, with the change of stress state embodying plastic accommodation. Second, the GS experiences successive layer-by-layer micro-yielding. This leads to an extended elasto-plastic transition at strains as large as $1 \%$. Third, strain localization occurs in the NS layer soon after yielding, as shown by the drastic decrease of $\varepsilon_{11}$. Strain softening thus occurs in the NS layer, in contrast to the long-held assumption that strain hardening occurs in the NS layer. Finally, plastic accommodation manifests as the change of stress state and induces heterogeneous deformation-induced hardening, which stabilizes an already unstable NS layer allowing the GS to continue to deform uniformly. The insights presented in this paper are helpful to tailor the engineering GSs for the extraordinary synergy between strength and ductility.

Received 4 September 2020; accepted 19 October 2020; published online 5 January 2021

1 Lu K. Making strong nanomaterials ductile with gradients. Science, 2014, 345: 1455-1456

2 Lu K. Stabilizing nanostructures in metals using grain and twin boundary architectures. Nat Rev Mater, 2016, 1: 16019

3 Fang TH, Li WL, Tao NR, et al. Revealing extraordinary intrinsic tensile plasticity in gradient nano-grained copper. Science, 2011, 331: $1587-1590$

4 Wu XL, Jiang P, Chen L, et al. Extraordinary strain hardening by gradient structure. Proc Natl Acad Sci USA, 2014, 111: 7197-7201

5 Cheng Z, Zhou H, Lu Q, et al. Extra strengthening and work hardening in gradient nanotwinned metals. Science, 2018, 362: eaau1925

6 Wu XL, Jiang P, Chen L, et al. Synergetic strengthening by gradient structure. Mater Res Lett, 2014, 2: 185-191

$7 \mathrm{Wu} \mathrm{X}$, Zhu Y. Heterogeneous materials: A new class of materials with unprecedented mechanical properties. Mater Res Lett, 2017, 5: 527-532

8 Zhu Y, Wu X. Perspective on hetero-deformation induced (HDI) hardening and back stress. Mater Res Lett, 2019, 7: 393-398

9 Hall EO. Variation of hardness of metals with grain size. Nature, 1954, 173: 948-949

10 Cracknell A, Petch NJ. Frictional forces on dislocation arrays at the lower yield point in iron. Acta Metall, 1955, 3: 186-189

11 Thilly L, Van Petegem S, Renault PO, et al. A new criterion for 
elasto-plastic transition in nanomaterials: Application to size and composite effects on $\mathrm{Cu}-\mathrm{Nb}$ nanocomposite wires. Acta Mater, 2009, 57: 3157-3169

12 Sinclair CW, Saada G, Embury JD. Role of internal stresses in codeformed two-phase materials. Philos Mag, 2006, 86: 4081-4098

13 Brandstetter S, Van Swygenhoven H, van Petegem S, et al. From micro- to macroplasticity. Adv Mater, 2006, 18: 1545-1548

$14 \mathrm{Wu} \mathrm{X}, \mathrm{Zhu} \mathrm{Y}, \mathrm{Lu} \mathrm{K}$. Ductility and strain hardening in gradient and lamellar structured materials. Scripta Mater, 2020, 186: 321-325

15 Ashby MF. The deformation of plastically non-homogeneous materials. Philos Mag-A J Theor Exp Appl Phys, 1970, 21: 399-424

16 Gao H, Huang Y, Nix WD, et al. Mechanism-based strain gradient plasticity? I. Theory. J Mech Phys Solids, 1999, 47: 1239-1263

17 Wei $\mathrm{Y}$, Wang $\mathrm{X}, \mathrm{Wu} \mathrm{X}$, et al. Theoretical and experimental researches of size effect in micro-indentation test. Sci China Ser AMath, 2001, 44: 74-82

18 Gao H, Huang Y. Geometrically necessary dislocation and sizedependent plasticity. Scripta Mater, 2003, 48: 113-118

19 Orowan E. Causes and effects of internal stresses. In: Rassweiler GM, Grube WL (eds.). Internal Stress and Fatigue in Metals. London: Elsevier, 1959, 59-80

20 Cottrell AH. Dislocations and Plastic Flow in Crystals. Oxford: Oxford University Press, 1953, 111-174

21 Gibeling JG, Nix WD. A numerical study of long range internal stresses associated with subgrain boundaries. Acta Metall, 1980, 28: $1743-1752$

22 Ibrahim N, Embury JD. The Bauschinger effect in single phase b.c.c. materials. Mater Sci Eng, 1975, 19: 147-149

23 Mughrabi $\mathrm{H}$. On the role of strain gradients and long-range internal stresses in the composite model of crystal plasticity. Mater Sci Eng-A, 2001, 317: 171-180

24 Yang M, Pan Y, Yuan F, et al. Back stress strengthening and strain hardening in gradient structure. Mater Res Lett, 2016, 4: 145-151

25 Yuan F, Yan D, Sun J, et al. Ductility by shear band delocalization in the nano-layer of gradient structure. Mater Res Lett, 2019, 7: 1217

26 Lee HH, Yoon JI, Park HK, et al. Unique microstructure and simultaneous enhancements of strength and ductility in gradientmicrostructured $\mathrm{Cu}$ sheet produced by single-roll angular-rolling. Acta Mater, 2019, 166: 638-649

27 Lin Y, Pan J, Zhou HF, et al. Mechanical properties and optimal grain size distribution profile of gradient grained nickel. Acta Mater, 2018, 153: 279-289

28 Zhou X, Li XY, Lu K. Strain hardening in gradient nano-grained $\mathrm{Cu}$ at $77 \mathrm{~K}$. Scripta Mater, 2018, 153: 6-9

29 Wang JJ, Tao NR, Lu K. Revealing the deformation mechanisms of nanograins in gradient nanostructured $\mathrm{Cu}$ and $\mathrm{CuAl}$ alloys under tension. Acta Mater, 2019, 180: 231-242

30 Zeng Z, Li X, Xu D, et al. Gradient plasticity in gradient nanograined metals. Extreme Mech Lett, 2016, 8: 213-219

31 Wang Y, Huang C, Li Y, et al. Dense dispersed shear bands in gradient-structured Ni. Int J Plast, 2020, 124: 186-198

$32 \mathrm{Li} \mathrm{J,} \mathrm{Lu} \mathrm{W,} \mathrm{Gibson} \mathrm{J,} \mathrm{et} \mathrm{al.} \mathrm{Compatible} \mathrm{deformation} \mathrm{and} \mathrm{extra}$ strengthening by heterogeneous nanolayer composites. Scripta Mater, 2020, 179: 30-35

33 Zhu L, Ruan H, Chen A, et al. Microstructures-based constitutive analysis for mechanical properties of gradient-nanostructured 304 stainless steels. Acta Mater, 2017, 128: 375-390

34 Cao R, Yu Q, Pan J, et al. On the exceptional damage-tolerance of gradient metallic materials. Mater Today, 2020, 32: 94-107
35 Cheng Z, Lu L. The effect of gradient order on mechanical behaviors of gradient nanotwinned Cu. Scripta Mater, 2019, 164: 130134

36 Zhu YT, Wu XL. Ductility and plasticity of nanostructured metals: Differences and issues. Mater Today Nano, 2018, 2: 15-20

37 Lu K, Lu J. Surface nanocrystallization (SNC) of metallic materialspresentation of the concept behind a new approach. J Mater Sci Technol, 1999, 15: 193-197

$38 \mathrm{Wu}$ X, Tao N, Hong Y, et al. Microstructure and evolution of mechanically-induced ultrafine grain in surface layer of Al-alloy subjected to USSP. Acta Mater, 2002, 50: 2075-2084

39 Jia N, Cong ZH, Sun X, et al. An in situ high-energy X-ray diffraction study of micromechanical behavior of multiple phases in advanced high-strength steels. Acta Mater, 2009, 57: 3965-3977

40 Cheng S, Wang YD, Choo $\mathrm{H}$, et al. An assessment of the contributing factors to the superior properties of a nanostructured steel using in situ high-energy X-ray diffraction. Acta Mater, 2010, 58: 2419-2429

41 Yang MX, Yuan FP, Xie QG, et al. Strain hardening in Fe-16Mn10Al-0.86C-5Ni high specific strength steel. Acta Mater, 2016, 109: 213-222

42 Bian X, Yuan F, Wu X, et al. The evolution of strain gradient and anisotropy in gradient-structured metal. Metall Mat Trans A, 2017, 48: 3951-3960

43 Bert CW, Mills EJ, Hyler WS. Effect of variation in Poisson's ratio on plastic tensile instability. J Basic Eng, 1967, 89: 35-39

44 Chaudhari P, Scattergood RO. On the pile-up model for yielding. Acta Metall, 1966, 14: 685-687

45 Wang MM, Tasan CC, Ponge D, et al. Nanolaminate transformation-induced plasticity-twinning-induced plasticity steel with dynamic strain partitioning and enhanced damage resistance. Acta Mater, 2015, 85: 216-228

46 Han Q, Asgari A, Hodgson PD, et al. Strain partitioning in dualphase steels containing tempered martensite. Mater Sci Eng-A, 2014, 611: 90-99

47 Wu X, Yang M, Yuan F, et al. Heterogeneous lamella structure unites ultrafine-grain strength with coarse-grain ductility. Proc Natl Acad Sci USA, 2015, 112: 14501-14505

48 Yang M, Yan D, Yuan F, et al. Dynamically reinforced heterogeneous grain structure prolongs ductility in a medium-entropy alloy with gigapascal yield strength. Proc Natl Acad Sci USA, 2018, 115: 7224-7229

49 Yang MX, Li RG, Jiang P, et al. Residual stress provides significant strengthening and ductility in gradient structured materials. Mater Res Lett, 2019, 7: 433-438

50 Xing YM, Lu J. An experimental study of residual stress induced by ultrasonic shot peening. J Mater Process Tech, 2004, 152: 56-61

51 Ortiz AL, Tian JW, Villegas JC, et al. Interrogation of the microstructure and residual stress of a nickel-base alloy subjected to surface severe plastic deformation. Acta Mater, 2008, 56: 413-426

52 Wilson DV, Bate PS. Influences of cell walls and grain boundaries on transient responses of an IF steel to changes in strain path. Acta Metall Mater, 1994, 42: 1099-1111

53 Wilson DV, Bate PS. Internal elastic strains in an IF steel following changes in strain path. Acta Mater, 1996, 44: 3371-3383

54 Schwab R, Ruff V. On the nature of the yield point phenomenon. Acta Mater, 2013, 61: 1798-1808

55 Wang YD, Peng RL, Almer J, et al. Grain-to-grain stress interactions in an electrodeposited iron coating. Adv Mater, 2005, 17: $1221-1226$ 
Champion Y, Langlois C, Guérin-Mailly S, et al. Near-perfect elastoplasticity in pure nanocrystalline copper. Science, 2003, 300: 310-311

57 Huang CX, Wang YF, Ma XL, et al. Interface affected zone for optimal strength and ductility in heterogeneous laminate. Mater Today, 2018, 21: 713-719

58 Xiang Y, Vlassak JJ. Bauschinger and size effects in thin-film plasticity. Acta Mater, 2006, 54: 5449-5460

59 Zhou H, Huang C, Sha X, et al. In-situ observation of dislocation dynamics near heterostructured interfaces. Mater Res Lett, 2019, 7: 376-382

60 Wang Y, Chen M, Zhou F, et al. High tensile ductility in a nanostructured metal. Nature, 2002, 419: 912-915

61 Liu XL, Xue QQ, Wang W, et al. Back-stress-induced strengthening and strain hardening in dual-phase steel. Materialia, 2019, 7: 100376

Acknowledgements This work was supported by the Ministry of Science and Technology, China (2017YFA0204402), NSFC Basic Science Center Program for "Multiscale Problems in Nonlinear Mechanics" (11988102), the National Natural Science Foundation of China (11972350 and 11890680), and the Chinese Academy of Sciences (XDB22040503).

Author contributions Wu X designed the research; Wu X, Wei Y, and Wang $\mathrm{Y}$ conceived the ideas; Yang M, Li R, Wang Y and Wu X conducted synchrotron measurements and analysis; Wu X conducted TEM observations; all authors contributed to data analysis and discussions; Wu X, Zhu Y, and Wei Y wrote the paper.

Conflict of interest The authors declare that they have no conflict of interest.

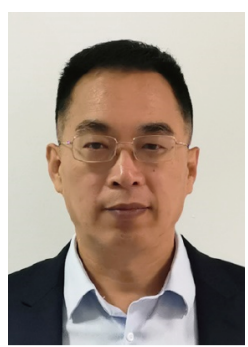

Xiaolei Wu has been a professor since 2004 at the State Key Laboratory of Nonlinear Mechanics, Institute of Mechanics, Chinese Academy of Sciences (CAS). He received his BS degree in 1986 in materials science from Taiyuan University of Technology, and PhD degree in 1995 from Northwestern Polytechnical University. His current research focuses on the research and understanding of the hetero-structure and deformation physics in advanced metallic materials for structural applications.

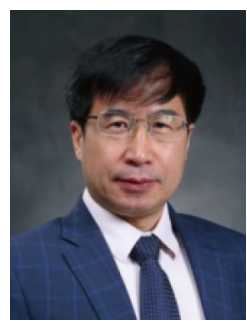

Yandong Wang received his $\mathrm{PhD}$ degree from the University of Science \& Technology Beijing (USTB). He is currently a Changjiang distinguished professor in the State Key Laboratory for Advanced Metals and Materials (USTB), with main research interest focused on the microstructural characterizations using synchrotron $\mathrm{X}$ ray diffraction and neutron scattering methods and developments of new-type shape memory alloys and high-strength alloys. He conducted his research as various research positions in universities and national laboratories in Europe and USA.

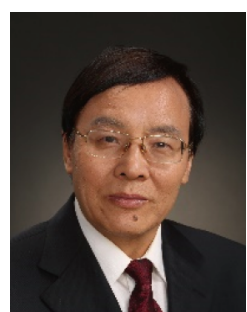

Yueguang Wei, Boya Chair Professor of Peking University and CAS member, graduated from Tsinghua University with a PhD degree of solid mechanics in 1992. He was a Post-doctoral Fellow (1995-1998) at Harvard University, and a professor (1998-2016) at the Institute of Mechanics of CAS. He joined Peking University as a Boya Chair Professor in November 2016. His main research interests include the trans-scale mechanics of solid, elastoplastic fracture mechanics, and composite mechanics.

\section{拉伸变形时梯度结构的塑性协调}

武晓雷 ${ }^{1,2^{*}}$, 杨沐釒金, 李润光 ${ }^{3}$, 姜萍 ${ }^{1}$, 袁福平 ${ }^{1,2}$, 王沿东 ${ }^{3 *}$, 朱运田 ${ }^{5}$, 魏悦广 广 $^{*}$

摘要 梯度结构是一种典型跨尺度微观结构, 其内部不同尺度晶粒 具有迥异的内禀塑性稳定性, 塑性协调是其获得优异强韧性的关 键微观机制. 本研究采用同步辐射原位拉伸测试方法, 对梯度结构 在拉伸变形过程中, 由表及里不同深度结构层的点阵应变演化进 行了研究. 结果表明, 梯度结构拉伸时具有两类塑性协调响应: 一 是由逐层微观屈服导致的瞬态弹-塑性变形, 使纳米结构层的弹-塑 性应变范围延后和扩展; 二是纳米结构表层的塑性局域化和软化. 梯度结构塑性协调引起了层间和层内应力状态的转变, 导致层间 微观力学行为响应的显著差异, 利用异质变形诱导应变硬化和林 位错硬化, 促使梯度结构中纳米结构表层获得与芯部粗晶层相当 的拉伸均匀应变. 